\section{GROUPCOM: VAX-based software for conducting small-group research}

\section{JOHN M. LEVINE, RAYMOND J. McKNIGHT, and STEPHANIE M. GREEN \\ University of Pittsburgh, Pittsburgh, Pennsylvania}

Levine and Stein (1978) described a computer program (GROUPCOM) for conducting on-line social psychological research. The original version of the program was written for the Experimental Time-Sharing System (ETSS) of the Learning Research and Development Center at the University of Pittsburgh (Fitzhugh \& Glaser, 1975). Because ETSS was a unique system, many potential users could not easily implement GROUPCOM. In order to make GROUPCOM available to more investigators, we have recently rewritten the program to run on a Digital Equipment Corporation (DEC) VAX-11/780 system. The purpose of this paper is to review briefly the capabilities of GROUPCOM and to describe how investigators can obtain the program and related documentation.

GROUPCOM is an extremely flexible program designed to facilitate research on a variety of social psychological phenomena, including social influence, social comparison, group decision making, impression management, and leadership. Among the advantages of the program are the following: Subjects can interact with either real or simulated others; data can be collected from several subjects at once; different subjects can be run simultaneously in different conditions; lengthy written messages, as well as brief responses, can be presented and collected; on each trial, the experimenter can control a number of important parameters (e.g., response order, response type, display format); and very little experimenter effort is required at run time to present experimental trials or to record subjects' responses.

Program Options. A given experimental condition can contain up to 200 "real" and/or "fake" trials, interspersed in any order. On real trials, two to six subjects can communicate with one another by entering responses on their terminal keyboards. These responses then appear on the screen of each terminal. On fake trials, one to six subjects can "communicate" with one to nine simulated others. After entering responses on their keyboards, subjects see predetermined responses allegedly entered by other (simulated) subjects. Because the information presented to each subject on fake trials is controlled independently, subjects can receive different

This work was supported by funds from the National Institute of Education to the Learning Research and Development Center at the University of Pittsburgh. Requests for reprints should be sent to John M. Levine, 711 LRDC Building, University of Pittsburgh, Pittsburgh, Pennsylvania 15260. simulated responses. On both real and fake trials, the experimenter determines whether subjects enter short answers (votes), long answers (notes), or both votes and notes.

When votes are collected, the experimenter also controls the form of the votes, the manner in which the votes are collected, and the manner in which the votes are displayed. Votes can be characters or integers within a predefined range, or free-formatted input up to 25 characters in length. Votes can be collected using either a sequential or a batch method. In the sequential method, each subject receives a cue when it is his or her turn to respond. In the batch method, all subjects are cued simultaneously and respond at will. Votes can be either displayed or not displayed to subjects. When displayed, votes collected using the sequential method are shown one at a time as they are obtained; votes collected using the batch method are held and then shown together after the last vote is obtained. On real trials, subjects see their own and other group members' actual votes. On fake trials, subjects see predetermined votes allegedly entered by simulated subjects. In addition, for their own vote on fake trials, subjects can see either the vote that they entered or a predetermined vote (e.g., a subject might receive the word "incorrect" after entering the answer to a problem). The experimenter controls the amount of time the votes are displayed, as well as the visual configuration of displayed votes (e.g., columns of $\mathrm{Xs}$ above numbers on a response continuum; a list of numbers, letters, words, or short statements identified by subject response number). In addition, on fake trials, the experimenter controls the latency preceding the appearance of each simulated vote. Options for votes can be changed from trial to trial.

When notes are collected, subjects are allowed to type free-formatted messages up to 10 lines in length. Notes can be either displayed or not displayed to subjects. On real trials, subjects see other group members' actual notes, identified by sender. The experimenter controls which notes each subject sees. On fake trials, subjects see predetermined notes, identified by simulated sender. The experimenter controls the content and alleged sender of each note. On both real and fake trials, the experimenter controls how long each note is shown. Options for notes can be changed from trial to trial.

Each subject is assigned to one of $n$ response positions on each trial. On real trials, where $n$ equals the number of actual subjects, each subject is assigned to a different response position (i.e., one subject is assigned to Position 1, another subject is assigned to Position 2, and so on). On fake trials, where $n$ equals the number of alleged subjects in the group, each subject can be assigned to any of the $n$ response positions (e.g., all subjects could be assigned to Position 3). Subjects' response positions can be altered from trial to trial. 
The sequencing of trials and the timing of events within trials can be controlled by either the experimenter (from a master terminal) or the program. Information about other subjects can be presented before and during the experimental trials, and a postexperimental questionnaire can be administered afterward. Subjects' responses and response latencies, as well as extensive information about trial type, responses seen by subjects, etc., are recorded on an output disk file.

Although this description of GROUPCOM highlights its utility for investigating social interaction, the program also can be used for other purposes. For example, each subject can be told that he or she is working independently and can be shown only his or her own answers. In this mode, GROUPCOM can be used to obtain responses from up to six subjects working simultaneously but independently.

Technical Information. The GROUPCOM program allows subjects and the experimenter to run as interrelated jobs in a time-sharing system. The GROUPCOM program consists of three program modules: COMPIL (compiler program), SUBJECT (subject program), and MASTER (master, or experimenter, program). One or more disk files are associated with each module. Several different file types are used in the GROUPCOM program. These file types, together with the program modules, constitute a program that allows a great deal of experimenter control and flexibility. The COMPIL, SUBJECT, and MASTER modules, as well as the interrelationships among these modules, are described in more detail by Levine and Stein (1978).

The GROUPCOM program, which contains approximately 3,000 noncomment statements, was written in standard VAX FORTRAN-77. Two differences between this and earlier versions of FORTRAN are (1) the CHARACTER data type and (2) the ability to support "structured" FORTRAN code. The CHARACTER facilities were used in the GROUPCOM program to ease the handling of textual data. Some "structured code" features were used to reduce conditional branch. ing and the number of statements, which in turn made the programs more efficient and the listings easier to read.

Two built-in special features of the VMS operating system were used in the GROUPCOM program. They are Common Event Flags (and the System Services to manipulate them) and System Common. The Event Flags are used as signaling devices between the MASTER and SUBJECT programs to synchronize their interaction. In general, the MASTER program sets an Event Flag to show that it is ready for a SUBJECT program to do something, and the SUBJECT program sets a corresponding flag when it has finished what it was signaled to do. The signaling interaction can become complex under some conditions, but it always remains essentially a "ready-complete" cycle. The System Common, a specially defined block of shared memory, is used to exchange text between the MASTER and SUBJECT programs. In some cases, MASTER sends text to the SUBJECT programs in a portion of the System Common block. In most MASTER-SUBJECT interactions, the SUBJECT programs send response text back to the MASTER program in their own portions of the System Common.

The GROUPCOM program is currently operating on a DEC VAX-11/780 computer using the VMS operating system. The hardware configuration of this VAX includes $4 \mathrm{MB}$ of main memory, two 67-MB and two 300-MB disk drives, one magnetic-tape drive, 56 serial lines for terminal communication, and several printers offering a variety of printout qualities and speeds. All of these features are not needed to run GROUPCOM; any VAX/VMS system compatible with the Model 780 and capable of supporting at least seven interactive users simultaneously can accommodate the GROUPCOM program.

GROUPCOM uses DEC VT52 or equivalent terminals for the MASTER and SUBJECT stations. These are video terminals that normally operate at a rate of 1200 baud (approximately 120 characters/sec). The baud rate has no bearing on the correct operation of the program, but it does affect the speed with which information is displayed on the terminal screen. We recommend a terminal baud rate of 1200 or higher for GROUPCOM.

Program Availability. A distribution kit is available for investigators interested in using GROUPCOM. The kit includes a nine-track magnetic tape and a printed installation guide, which describes how to install GROUPCOM on a VAX system. The tape contains source files and extensive documentation files. Documentation files clarify the steps necessary to set up and run experiments using GROUPCOM. In addition, a practice experiment that demonstrates many of the program's capabilities is included. This 20-trial experiment, designed for four subjects, illustrates various types of real and fake trials that can be run using GROUPCOM. All files necessary to run the practice experiment are provided on the tape. The tape and installation guide are available for $\$ 85.00$ to cover the costs of purchasing, preparing, and mailing the tape.

\section{REFERENCES}

Fitzhugh, R. J., \& Glaser, R. A general purpose computer system for a research and development center. American Psychologist, 1975, 30, 219-225.

LEvine, J. M., \& STEIN, H. GROUPCOM: A computer program for investigating social processes in small groups. Behavior Research Methods \& Instrumentation, 1978, 10, 191-195.

(Manuscript accepted for publication October 31, 1983.) 